\title{
The Population Growth of a Rotifer Brachionus plicatilis and Life History of Amictic Females
}

\author{
Hisashi Kurokura, ${ }^{* 1}$ Maria Elena Castellanos Paez, ${ }^{* 2}$ \\ and Shogoro Kasahara*2
}

(Received May 23, 1990)

\begin{abstract}
The relation between the individual life history characteristics and the population growth in a rotifer Brachionus plicatilis is discussed. The population growth rates $(r)$ in three different strains of the rotifer calculated theoretically from observed individual life history characteristics, embryonic developmental time, duration of pre-reproductive, reproductive and post-reproductive phase, and number of offspring, is presented. The observed characteristics varied between different strains. Due to these differences, the calculated population growth rate between 15 days and 20 days after the inoculation of one freshly laid egg varied from 0.30 to 0.46 . The major factors which influence the population growth rate seem to be spawning interval. Increasing the number of offspring from one individals and the number of reproductive individuals would not bring about the desired increase in the population growth rate as well as the shortening of developmental time and spawning interval.
\end{abstract}

Population growth and fitness to the environment are generally expressed through the demographic parameter $r$, the intrinsic rate of increase. The value of $r$ is determined by several characteristics in the life history of individuals making up the population. The effect of the net reproductive rate $(R o)$, developmental time and generation time have been reported in previous works. ${ }^{1,2)}$

There are several methods of estimating the value of $r$, in which a stable age distribution is assumed. However, in practice the situation may be different in particular, the percentage of reproductive individuals is not constant in early phases of population growth. This is due to the relatively small nmber of individuals inoculated into a broad volume of environmental space.

A typical example of the above can be seen in the population of a rotifer Brachionus plicatilis which is widely used as a food organism in fish seedling production. Studies on the rotifer in relation to aquaculture have so far emphasized their biology, rearing techniques and nutritional value. ${ }^{3,4)}$ The existence of various strains in B. plicatilis have been reported. These strains differed from each other in population growth as well as in morphological characteristics. ${ }^{5,8)}$
B. plicatilis can reproduce parthenogenetically and the frequency of bisexual reproduction is quite low in some strains. This means that the genetical variation within a rotifer population in the growth phase is quite limited and such a population is similar to the monoclonal population. Through experimental studies on the relationship between the population growth and individual life history it will be possible to clarify the contribution of each factor to population growth. As the first step of a series of studies, we examined the life history of amictic females from three different stratins of $B$. plicatilis and compared their reproductive features by means of individual rearing experiments under similar environmental conditions. From these results, we simulated the population growths by employing an equation which does not require a stabel age distribution.

\section{Materials and Methods}

The three strains of $B$. plicatilis used in the present work were derived from cultures which had been maintained under laboratory conditions for several years and kindly supplied by K. Hirayama, Nagasaki University (Nagasaki and Thai

*1 Fisheries Laboratory, The University of Tokyo, Maisaka, Maisaka, Hamana, Shizuoka 431-02, Japan

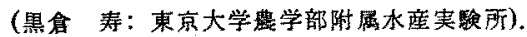

*2 Aquaculture Laboratory, Faculty of Applied Biological Science, Hiroshima University, Higashi-Hiroshima 720, Japan (M. E. Castellanos Paez, 笠原正五郎：広島大学生物生座学部)。 
Table 1. Sources and sizes of adult females of Brachionus plicatilis used in the present study

\begin{tabular}{llc}
\hline Strain & Collection site & $\begin{array}{c}\text { Average lorica } \\
\text { length } \\
\pm \mathrm{SD}(\mu \mathrm{m})\end{array}$ \\
\hline Thai & Thailand & $155.5 \pm 8.1$ \\
Yashima & Kagawa, Japan & $201.3 \pm 16.8$ \\
Nagasaki & Nagasaki, Japan & $237.4 \pm 8.9$ \\
\hline
\end{tabular}

strains) and S. Yamashita and H. Hirata, Kagoshima University (Yashima strain). Table 1 shows the collection site and mean lorical length of adult females belonging to each strain.

\section{Embryonic Developmental Time}

Stock culture of these three strains were fed with Nannochloropsis sp, and maintained under constant environmental conditions (temperature: $25^{\circ} \mathrm{C}$, salinity: $25 \%$ L. D. 12:12), Egg-carrying amictic females were individually transferred using a micropipet from a stock culture into wells of a Corning 25820 multiwell plate containing $1 \mathrm{ml}$ of a suspension of Nannochloropsis $s p$. $\left(2.77 \times 10^{6}\right)$. Neonate females produced during the $24 \mathrm{~h}$ of incubation were individually placed into new wells with fresh medium, and $24 \mathrm{~h}$ later the females with fully developed ova were individually transferred onto a glass cavity slide containing $1 \mathrm{ml}$ of medium. Each slide wes placed in a Petri dish to avoid evaporation. They were observed under a compound light microscope every 30 min until the first egg was laid. These eggs were subsequently observed at $2 \mathrm{~h}$ intervals, but during the last $2 \mathrm{~h}$ before hatching, when the eye, cilia on the corona and mastax movement are recognizable, ${ }_{3}$ ) observations were performed more frequently (30 min interval). The duration between the spawning of the egg and the hatching were recorded as the embryo developmental time $(e)$.

\section{Reproduction and Life Span}

A hundred amictic females from each stock culture were separately transferred into Erlenmeyer flasks, each containing $100 \mathrm{ml}$ of medium, and allowed to grow for 3 days, this was perfromed so as to acclimatize the individuals to to the environmental condition mentioned above. From these cultures another 100 females were then transferred to a freshly prepared medium to repeat the same procedure. After another 3 days when the population was in an exponential growth phase, ovigetous females were individually transferred into wells of a multiwell plate containing $1 \mathrm{ml}$ of medium. During $24 \mathrm{~h}$ of incubation, neonate females were produced and were individually placed into new wells with fresh medium for an additional $24 \mathrm{~h}$, during which tine the animals matured and laid their first egg. They were transferred again into new wells with freshly prepared medium. In the following $24 \mathrm{~h}$, hatching neonates from the first egg were placed individually into hand made test tubes (48 $\mathrm{mm}$ in length and $4.5 \mathrm{~mm}$ in diameter), each containing $0.25 \mathrm{ml}$ of medium. They were transferred daily into clean test tubes containing fresh medium, and at each transfer, the newly laid eggs were counted and the neonates produced were removed. This procedure was repeated for more than 50 individuals for each strain until they died. In order to assess the differences in life history among strains, the following parameters were determined, $a$ ) average duration of pre-reproductive period; $b$ ) average duration of reproductive period; $c$ ) average duration of postreproductive period; $n$ ) average number of offspring; net repeoductive rate $(R o)$. From these parameters, we simulated growth curve and population age structure thorough the counting by following method.

In the case, $t=0$ is the moment when a freshly laid egg are inoclated. The number of individuals older than age $A g$ at time $t$ is expressed as follows.

$$
\begin{aligned}
& N(T)=N(T-1)+r(T))-r(T-L+A) \\
& r(T)=r(T-B-h) \\
& N(j)=0, \quad r(j)=0, \quad r(0)=1, j<0
\end{aligned}
$$

where

$N(T)$ : Number of indivivulas older than age $A g$ at time

$$
t=T I+A
$$

$r(T)$ : Number of indivivuals reached at age $A g$ at time

$$
t=T I+A
$$

$I: \quad$ spawning interval $=b /(n-1)$,

$$
\begin{aligned}
& B=(e+a) / I, \quad A=A g / l, \\
& L=(e+a+b+c) / I
\end{aligned}
$$

$a$ : duration of pre-reproductive phase

$b$ : duration of reproductive phase

$c$ : duration of post-reproductive phase.

$e$ : embryonic developmental tim

From Formula (1)

$$
N(T)=R(T)-R(T-L+A)
$$

here, 


$$
R(T)=\sum_{i=0}^{T} r(i)
$$

The general terms of $r(T)$ and $R(T)$ can be expressed as follows:

$$
r(T)=\sum_{g=1}^{\left[\frac{T}{g}\right]\left[\frac{T-g B}{\sum_{k=1}^{n}}\right]}(-1)^{k}{ }_{0} C_{k} \cdot{ }_{T-\vartheta B-k n+g-1} C_{\sigma-1}
$$

and

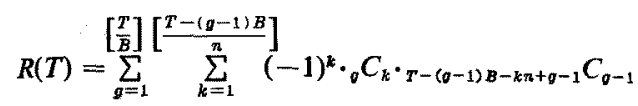

In present paper, the values of $N(T)$ were calculated using Formula (6).

The value of $r$ was calculated from the number of individuals at 15 and 20 days after the inoculation through the exponential equation,

$$
N_{t}=N_{0} \mathrm{e}^{r t} \text {. }
$$

\section{Results}

Obtained characteristics in life history of each strain are summarized in Table 2. Embryonic developmental time of the first eggs of amictic females varied from $16.5 \pm 0.7$ (mean + S. D.) to $20.4 \pm 0.9 \mathrm{~h}$, depending on the strain. The Thai strain had the shortest embryonic developmental time, followed by the Yashima and Nagasaki strain. The average life span varied from $8.9 \pm$ 2.6 to $13.2 \pm 2.4$ days, and the average number of offspring varied from 4.7 in Thai strain to 9.7 in Yashima strain. A wide range of variation in the number of offspring per female was shown by all the strains. Thai $(0-12)$; Yashima $(0-20)$ and

Table 2. Growth characteristics of each strain

\begin{tabular}{lccc}
\hline \multicolumn{1}{c}{ Strain } & Thai & Yashima & Nagasaki \\
\hline Embryonic developmental time (h) & $16.5 \pm 0.9$ & $18.8 \pm 1.1$ & $20.4 \pm 0.9$ \\
Pre-reproductive phase (day) & $2.2 \pm 0.7$ & $1.7 \pm 0.8$ & $2.4 \pm 0.6$ \\
Reproductive phase (day) & $5.5 \pm 2.6$ & $7.3 \pm 3.1$ & $7.8 \pm 2.3$ \\
Post-reproductive phase (day) & $1.1 \pm 1.0$ & $1.0 \pm 1.3$ & $2.9 \pm 1.8$ \\
Number of offspring & $4.7 \pm 3.2$ & $9.7 \pm 5.0$ & $6.0 \pm 1.9$ \\
Spawning interval (day) & 1.49 & 0.84 & 1.56 \\
$N_{z 0}$ & 398 & 4134 & 225 \\
$r$ & 0.33 & 0.46 & 0.30 \\
Reproductive individuals (\%) & 43 & 45 & 45 \\
\hline
\end{tabular}

\begin{tabular}{|c|c|c|c|c|c|}
\hline Characteristic & ss & df & mss & F & $\mathbf{P}$ \\
\hline \multicolumn{6}{|c|}{ Embryonic developmental time } \\
\hline total & 125.6 & 34 & & & \\
\hline strain & 96.4 & 2 & 48.2 & 52.7 & $<0.001$ \\
\hline residuals & 29.2 & 32 & 0.913 & & \\
\hline \multicolumn{6}{|c|}{ Pre-reproductive phase } \\
\hline total & 114.6 & 191 & & & \\
\hline strain & 17.2 & 2 & 8.60 & 16.7 & $<0.001$ \\
\hline residuals & 97.4 & 189 & 0.515 & & \\
\hline \multicolumn{6}{|c|}{ Reproductive phase } \\
\hline total & 1596.6 & 191 & & & \\
\hline strain & 177.3 & 2 & 88.7 & 11.8 & $<0.001$ \\
\hline residuals & 1419.3 & 189 & 7.51 & & \\
\hline \multicolumn{6}{|c|}{ Post-reproductive phase } \\
\hline total & 486.5 & 191 & & & \\
\hline strain & 130.8 & 2 & 65.4 & 34.8 & $<0.001$ \\
\hline residuals & 355.7 & 189 & 1.88 & & \\
\hline \multicolumn{6}{|c|}{ Number of offsprings } \\
\hline total & 3665.0 & 191 & & & \\
\hline strain & 945.0 & 2 & 472.5 & 32.8 & $<0.001$ \\
\hline residulas & 2720.0 & 189 & 14.4 & & \\
\hline
\end{tabular}

$N_{20}$ : number of individuals on the 20th day.

$r$ : growth rate between 15 th and 20 th day.

Table 3. ANOVA of each growth characteristic 
Table 4. Theoretical growth characteristics of several model case

\begin{tabular}{lccccc}
\hline \multicolumn{1}{c}{ case } & Thai & Yashima & case 1 & case 2 & case 3 \\
\hline $\begin{array}{l}\text { embryonic developmental } \\
\text { time (h) }\end{array}$ & 16.5 & 18.8 & 16.5 & 16.5 & $18.8^{*}$ \\
$\begin{array}{l}\text { pre-reproductive phase } \\
\text { (day) }\end{array}$ & 2.2 & 1.7 & 2.2 & 2.2 & $1.7^{*}$ \\
$\begin{array}{l}\text { reproductive phase (day) } \\
\text { post-reproductive phase }\end{array}$ & 5.5 & 7.3 & $13.0^{*}$ & $3.1^{*}$ & 5.5 \\
$\quad$ (day) & 1.1 & 1.0 & 1.1 & 1.1 & 1.1 \\
number of offspring & 4.7 & 9.7 & $9.7^{*}$ & 4.7 & 4.7 \\
spawning interval & 1.49 & 0.84 & 1.49 & 0.84 & 1.49 \\
$N_{20}$ & 398 & 4134 & 413 & 1817 & 710 \\
$r$ & 0.33 & 0.46 & 0.33 & 0.42 & 0.38 \\
Reproductive individuals & 43 & 45 & 48 & 31 & 49 \\
\hline$N_{20}:$ number of individuals on the 20th day. & & & & \\
$\quad$ r: growth rate between isth and 20th day. & & & &
\end{tabular}

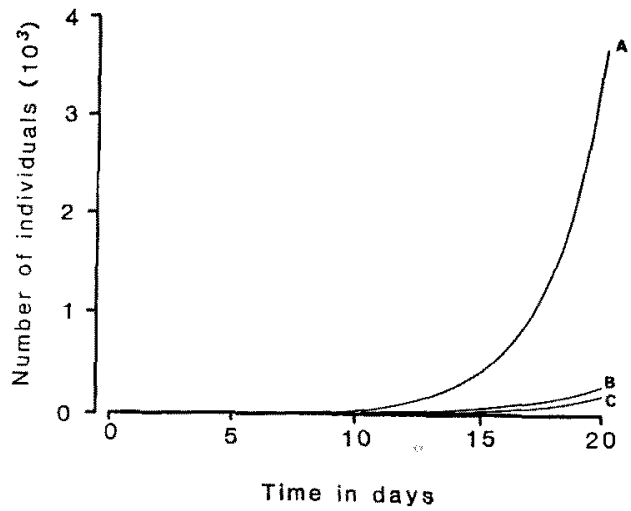

Fig. 1. Theoretical growth curve calculated from the individual life history of amictic female of three different strains of the rotifer. A: Thai strain, B: Yashima strain, C: Nagasaki strain.

Nagasaki (1-11). Individual life span in all the strains studied also showed a wide range of variation, Thai (4-15 days); Yashima (3-18 days) and Nagasaki (8-19 days). ANOVA revealed that there were highly significant difference among the mean values of each parameter for these three strains (Table 3).

Among the three different strains, the population of the Yashima strain can theoretically reach 4134 individuals 20 days after the inoculation of one freshly laid egg. On the other hand, populations of Thai and Nagasaki strains can only reach 398 and 225 individuals during the same period (Fig. 1). The $r$ was $0.33,0.46$, and 0.30 in Thai, Yashima and Nagasaki strains, respectively: Table 4 shows the results of the transposing some of the Yashima strain characters to the Thai strain. In Case 1, in which the number of offspring, 4.7, was replaced without a change in the spawning interval, the reproductive phase was prolonged to 13.0 days, though the value of $r$ was improved insignificantly. In case 2, where the spawing interval was changed without the changing the number of offspring, the reproductive phase was decreased from 5.5 to 3.1 days. However, the value of $r$ was increased from 0.33 to 0.42 . In the third case, the development time which consist of embryonic developmental time and pre-reproductive phase was shortened to 2.48 and this resulted in an $r$ value of 0.38 (Table 4). Among the above 3 cases, the shortening of the spawning interval produced the largest gain in $r$ value.

\section{Discussion}

Ruttner-Kolisko ${ }^{8)}$ reported that the developmental time of amictic eggs of $B$. plicatilis varied from $14 \mathrm{hr}$ at $25^{\circ} \mathrm{C}$ to $32 \mathrm{hr}$ at $15^{\circ} \mathrm{C}$. Yúfera ${ }^{8)}$ and Yúfera and Pasucual ${ }^{10}$ examined the effect of various factors on egg developmental time for two strains of $B$. plicatilis, and reported values from 15.53 to $25.57 \mathrm{~h}$. Ruttner-Kolisko ${ }^{8}$ ) reported that at $25^{\circ} \mathrm{C}$, the life time was 7 days, the average juvenile period 1.25 days, the average reproductive period 3.03 days, and the average senile period 2.12 days. In comparison, the present work showed that the life span, pre-reproductive and reproductive period for all three strains are longer. Only the post-reproductive period of the Nagasaki strain was similar to that observed by RuttnerKolisko. ${ }^{8)}$ The numbers of offspring obtained in the present study were smaller, when compared 
to those of previous work. Rumengan and Hirayama $^{\theta)}$ obtained more than 15 offspring at $25^{\circ} \mathrm{C}$ for 13 strains of $B$. plicatilis in which the three stratins used in the present work were included. In the present study, there were wide variations in the number of offspring, which range from 0 to 20 . This can be explained by the fact that the number of offspring is influenced by environmental conditions as well as the genotype. In this study, we did not performe any agitation to prevent precipitation of the algal suspension and this may be the one reason for the wide range and lower number of offspring than that observed by Rumengan and Hirayama. ${ }^{\text {) }}$ The data provided by the present study were obtained under environmental conditions which are probably not optimal for all the strains studied. The results of ANOVA shows clear differences among three strains with respect to each item (Table 3). Accrdingly, the values of each items can be regarded as strain specific characteristics.

A major premise of the equation used in the simulation of growth is the stability of the individual characters, implying that the characters are not changed either with age of individual or with generation. This premise also includes the hypothesis that the spawning interval is stable throughout the reproductive phase. Our results were obtained by using only the primiparous eggs of maternal rotifers. Piavaux ${ }^{11}$ has observed that the duration of embryonic development in some rotifers is affected by the age of the female. In our preliminary experiment, there was no apparent influence of age and generation on the characters observed in this study, and no distinct trend was observed in daily fecundity during the reproductive phase. Thus, this premise is not unrealistic in the case of $B$. plicatilis.

In order to evaluate the potential changes in the $r$ value that can be obtained by improvement of each parameter, we transpose some of the Yashima strain characters to Thai strain. In the case of decreasing spawning interval (case II), in which the number offspring remained the same as the Thai strain, the $r$ value increases to 0.42 . When the number of offspring is increased in the simulation (case I), the obtainable $r$ value remained lower than case 2, case, 3 . This clearly shows the positive effect of a short spawning interval. Similar theoretical results were also discussed in relation to population which has stable age structure by Snell. ${ }^{12}$
Strains of $B$. plicatilis differ from each other morphologically as well as with respect to life history traits. Life history has a close relation with morphology, nutritional requirement and temperature. As the present experiments were not performed under optimum conditions for each strain and we do not have any reliable data concerning the relationships involved. However, in other strains of planktonic rotifer, curvilinear relationship which could be described by Belegradek's equation was reported between temperature and developmental time. ${ }^{12)}$ The growth rate of so-called S-type strains of $B$. plicatilitis, in which egg size and lorica length of adult female are smaller than those of L-type strains, is higher than L-type strains at higher temperatures. It is reported to be higher at $34^{\circ} \mathrm{C}$ than at $24^{\circ} \mathrm{C}$, though the number of offspring $\left(R o\right.$ ) is smaller at $34^{\circ} \mathrm{C}^{8}$ ) Higher growth rate of the population in S-type strains at higher temperature are supposed to be achieved by a shorter embryonic developmental time and spawning interval. A temperature of $25^{\circ} \mathrm{C}$ is supposed to be far lower than the optimal temperature for Thai strain.

Another possible way of improving $r$ value is to increase the number of offspring. This can be achieved by optimizing the food supple level or decreasing energy requirement for oogenesis. However, increasing the number of offspring will not enhance the $r$ value as effectively as shortening the developmental time and spawning interval in the species which has large number of offspring. This strategy is effective only for the population which has very small number of offspring as reported by Snell."

Another important point which is revealed by the simulation model is that the percentages of reproductive individuals are smaller in case 2 than in case 1 or the original Thai strain, although the growth rate is higher in case 2 . Higher percentages of reproductive individuals does not always mean a higher population growth rate.

Since the characteristics of the life cycle pattern are influenced by the population density, the usefulness of the method used in present study is limited to the early phase of population growth. Validity for populations of different densities will require study by comparison between the actual population growth curve and theoretical growth of various strains of $B$. plicatilis. This study will enable a more precise assessment to be made of each parameter of the life cycle patterns 
on population growth.

\section{Acknowledgments}

We express our thanks to Mr. Tatsuro Akamine, Japan Sea Regional Fisheries Reseach Laboratory, and Dr. Yoshiharu Matsumiya, Faculty of Bioresources, Mie University for thier helpful suggestions in the mathematical presentations.

\section{References}

1) T. W. Snell: Oecologia, 32, 119-125 (1978).

2) A. Meats: Oecologia, 6, 223-237 (1971).

3) H. Hirata: Min. Rev. Data File Fish. Res., 1, 21-46 (1980).
4) E. Lubzens: Hydrobiologia, 147, 245-255 (1987).

5) S. Ito, H. Sakamoto, M. Hori, and K. Hirayama: Bull. Fac. Fish. Nagasaki Univ., 51, 9-16 (1981).

6) I. F. M. Rumengan and K. Hirayama: Proc. Second Asian Fish. Forum, Tokyo, Japan, 33-36 (1989).

7) M. E. Castellanos Paez, H. Kurokura, and S. Kasahara: J. Fac. Appl. Biol. Sci., Hiroshima Univ., 27, 93-99 (1988).

8) Ruttner-Kolisko: Dt. Zool, Ges., 65, 89-96 (1972).

9) M. Yúfera: Hydrobiologia, 147, 319-322 (1987).

10) M. Yúfera and E. Pasucual: Inv. Pesq., 48, 549-556 (1984).

11) A. Piavaux: Annls. Soc. Roy. Zool. Belg., 9, 237-247 (1970).

12) A. Herzig: Hydrobiologia, 104, 237-246 (1983). 